\title{
Full-thickness resection device (FTRD) for treatment of upper gastrointestinal tract lesions: the first international experience $\nabla$
}

다 (i) $(-)$

\author{
Authors \\ Kaveh Hajifathalian ${ }^{1}$, Yervant Ichkhanian², Qais Dawod ${ }^{1}$, Alexander Meining ${ }^{3}$, Arthur Schmidt ${ }^{4}$, Nicholas Glaser ${ }^{4}$, \\ Kia Vosoughi², David L. Diehl ${ }^{3}$, Ian S. Grimm ${ }^{5}$, Theodore James ${ }^{6}$, Adam W. Templeton ${ }^{7}$, Jason B. Samarasena ${ }^{8}$, Nabil \\ El Hage Chehade ${ }^{8}$, John G. Lee ${ }^{8}$, Kenneth J. Chang ${ }^{8}$, Meir Mizrahi ${ }^{9}$, Mohammed Barawi ${ }^{10}$, Shayan Irani ${ }^{11}$, Shai \\ Friedland ${ }^{12}$, Paul Korc ${ }^{13}$, Abdul Aziz Aadam ${ }^{14}$, Mohammad Al-Haddad ${ }^{15}$, Thomas E. Kowalski ${ }^{16}$, George Smallfield ${ }^{17}$, \\ Gregory G. Ginsberg ${ }^{18}$, Norio Fukami ${ }^{19}$, Michael Lajin ${ }^{20}$, Nikhil A. Kumta ${ }^{21}$, Shou-jiang Tang ${ }^{22}$, Yehia Naga ${ }^{22}$, Stuart K. \\ Amateau $^{23}$, Franklin Kasmin ${ }^{24}$, Martin Goetz ${ }^{25}$, Stefan Seewald ${ }^{26}$, Vivek Kumbhari², Saowanee Ngamruengphong ${ }^{2}$,

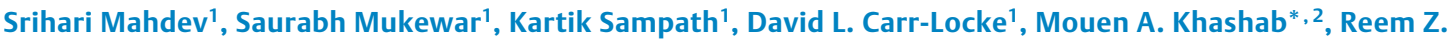 \\ Sharaiha*, 1
}

Institutions

1 Weill Cornell Medicine, Division of Gastroenterology and Hepatology, Department of Medicine, New York, NY

2 Division of Gastroenterology, Johns Hopkins Hospital, Baltimore, Maryland, United States

3 Interventional and Experimental Endoscopy, Department of Internal Medicine I, Ulm University, Ulm, Germany

4 Department of Medicine II, Medical Center, University of Freiburg, Faculty of Medicine, Freiburg, Germany

5 Department of Gastroenterology and Nutrition, Geisinger Medical Center, Danville, Pennsylvania, United States

6 Division of Gastroenterology and Hepatology, University of North Carolina, Chapel Hill, North Carolina, United States

7 Department of Gastroenterology, University of Washington, Seattle, Washington, United States

8 H. H. Chao Comprehensive Digestive Disease Center, Division of Gastroenterology and Hepatology, University of California, Irvine, Orange, California, United States

9 Department of Internal Medicine, Division of Gastroenterology, Center for Advanced Endoscopy, University of South Alabama, Mobile, Alabama, United States

10 Division of Gastroenterology and Hepatology, Ascension St. John hospital, Detroit, Michigan, United States

11 Digestive Disease Institute, Virginia Mason Medical Center, Seattle, Washington, United Stats

12 Division of Gastroenterology and Hepatology, Stanford University School of Medicine, Stanford, California, United States

\footnotetext{
* These authors contributed equally.
}

13 Department of Medicine, Division of Gastroenterology, Hoag Hospital, Newport Beach, California, United States

14 Division of Gastroenterology, Northwestern University, Chicago, Illinois, United States

15 Indiana University School of Medicine, Department of Medicine, Division of Gastroenterology, Indianapolis, Indiana, United States

16 Thomas Jefferson University, Philadelphia, Pennsylvania, United States

17 Division of Gastroenterology, Hepatology and Nutrition, Virginia Commonwealth University, Richmond, Virginia United States

18 Gastroenterology Division, University of Pennsylvania, Perelman School of Medicine, Philadelphia, Pennsylvania, United States

19 Division of Gastroenterology and Hepatology, Mayo Clinic Arizona, Scottsdale, Arizona, United States

20 SHARP Grossmont Hospital, La Mesa, California, United States

21 Dr. Henry D. Janowitz Division of Gastroenterology, Icahn School of Medicine at Mount Sinai, New York, New York, United States

22 Division of Digestive Diseases, Department of Medicine, University of Mississippi Medical Center, Jackson, Mississippi, United States

23 Division of Gastroenterology, University of Minnesota, Minneapolis, Minnesota, United States

24 Division of Gastroenterology, Lenox Hill Hospital, Northwell Health, New York, New York, United States

25 Innere Medizin I, Universitätsklinikum Tübingen, Tuebingen, Germany

26 Centre of Gastroenterology, Klinik Hirslanden, Zurich, Switzerland

submitted 3.3.2020

accepted after revision 29.6.2020 


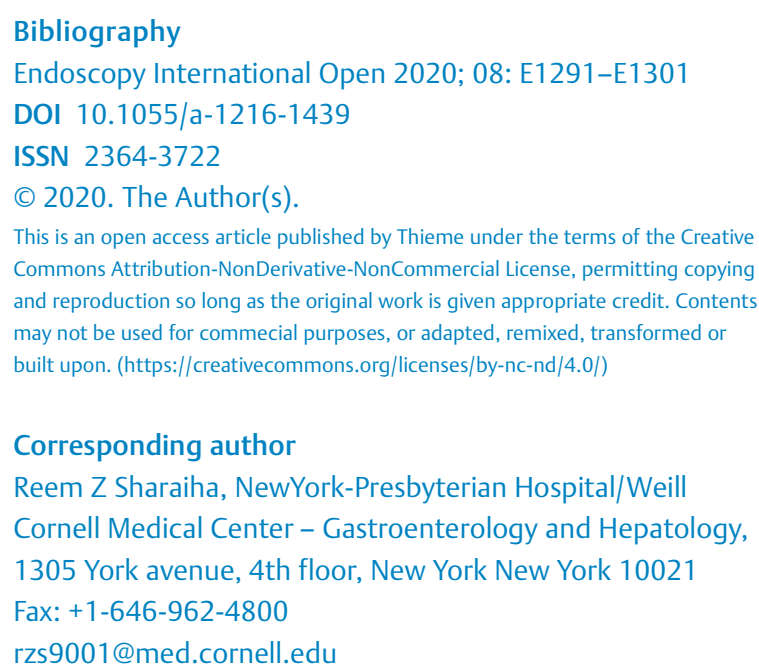

Corresponding author

Reem Z Sharaiha, NewYork-Presbyterian Hospital/Weill

Cornell Medical Center - Gastroenterology and Hepatology,

1305 York avenue, 4th floor, New York New York 10021

Fax: +1-646-962-4800

rzs9001@med.cornell.edu

\section{ABSTRACT}

Background and study aims The Full-Thickness Resection Device (FTRD) provides a novel treatment option for lesions not amenable to conventional endoscopic resection techniques. There are limited data on the efficacy and safety of FTRD for resection of upper gastrointestinal tract (GIT) lesions.
Patients and methods This was an international multicenter retrospective study, including patients who had an endoscopic resection of an upper GIT lesion using the FTRD between January 2017 and February 2019.

Results Fifty-six patients from 13 centers were included. The most common lesions were mesenchymal neoplasms $(n=23,41 \%)$, adenomas $(n=7,13 \%)$, and hamartomas ( $n$ $=6,11 \%$ ). Eighty-four percent of lesions were located in the stomach, and $14 \%$ in the duodenum. The average size of lesions was $14 \mathrm{~mm}$ (range 3 to $33 \mathrm{~mm}$ ). Deployment of the FTRD was technically successful in $93 \%$ of patients $(n=$ 52 ) leading to complete and partial resection in 43 (77\%) and $9(16 \%)$ patients, respectively. Overall, the FTRD led to negative histological margins (R0 resection) in $38(68 \%)$ of patients. A total of $12(21 \%)$ mild or moderate adverse events (AEs) were reported. Follow-up endoscopy was performed in 31 patients (55\%), on average 88 days after the procedure (IQR 68-138 days). Of these, 30 patients (97\%) did not have any residual or recurrent lesion on endoscopic examination and biopsy, with residual adenoma in one patient (3\%).

Conclusions Our results suggest a high technical success rate and an acceptable histologically complete resection rate, with a low risk of AEs and early recurrence for FTRD resection of upper GIT lesions.

\section{Introduction}

Beyond simple polypectomy, endoscopic resection of upper gastrointestinal tract (UGIT) mucosal and submucosal lesions is conventionally done using either endoscopic mucosal resection (EMR) or endoscopic submucosal dissection (ESD), and less frequently by submucosal tunneling endoscopic resection (STER) or endoscopic full-thickness resection (EFTR) [1]. With appropriate case selection, these techniques are associated with comparable outcomes and lower morbidity when compared to surgical resection [2,3]. Compared with EMR, ESD results in higher en-bloc and R0 (complete resection) rates achieving higher curative resection with lower recurrence rates [4]. However, ESD is associated with higher rates of adverse events (AEs) including bleeding and perforation, particularly with duodenal lesions, is technically complex, and takes longer to perform. In addition, ESD becomes technically challenging in cases where prior treatment leads to submucosal fibrosis.

EFTR using the Full-Thickness Resection Device (FTRD; Ovesco Endoscopy, Tübingen, Germany) is a novel method for resection of mucosal and submucosal lesions less than $3 \mathrm{~cm}$ in size. The FTRD consists of a special over-the -scope clip (OTSC) with an integrated snare and a separate tissue grasper designed for achieving a non-exposure full-thickness resection of the target lesion. Compared with conventional endoscopic resection techniques, the FTRD has potential for decreasing the procedure time and rate of complications, especially perforation, as well as providing an option for resection of non-lifting or previously incompletely treated lesions. Published data on use of the FTRD for colonic lesions show a favorable safety profile and efficacy [5-8], but the available evidence for its use in UGIT is limited to small single-center case series $[9,10]$. We present the first international multicenter study of safety and efficacy of FTRD for resection of UGIT lesions.

\section{Patients and methods}

This was an international multicenter retrospective study, including patients who had endoscopic resection of an UGIT lesion using the FTRD. Collaborators were invited to share their data on the safety and efficacy of using FTRD for UGIT lesions. Data from procedures performed between January 2017 and February 2019 were included in the study. For all procedures, information was provided to patients regarding FTRD procedure as a new option for management of their problem, its possible harms and potential benefits (e. g. lower expected risk of perforation, and shorter duration of procedure, potential to avoid regular surveillance procedures, or surgery), and alternative treatment options (e.g. ESD, or a surveillance program). Subsequently, written informed consent was obtained from all patients. This study followed the guidelines of the 1975 Declaration of Helsinki. The study protocol was reviewed and approved by the institutional review board at each study center, as well as the study coordinating center (IRBs 1804019146; 1701017930). 


\section{Outcomes}

The primary endpoints of this study were:

1. Technical success: Complete success was defined as reaching the target lesion with the FTRD, correct application of the FTRD clip on the lesion and immediate resection with the integrated snare and complete en bloc endoscopic resection. Complete en bloc endoscopic resection was defined as absence of macroscopic evidence of residual lesion after FTRD use as judged by the endoscopist. Partial success was defined as reaching the target lesion with the FTRD, correct application of the FTRD clip on the lesion and immediate resection with the integrated snare but partial endoscopic resection with positive macroscopic margins $[5,10]$. The procedure was considered incomplete if the target lesion could not be reached with the FTRD device or if the target lesion was reached but the endoscopist decided against deploying the FTRD due to lesion characteristics (e.g. device could not be oriented properly due to the anatomy, the lesion could not be accommodated in the cap, or the lesion could not be retracted with tissue grasper). Device failure was defined as over-the-scope clip (OTSC), tissue grasper, or integrated snare malfunction in any way leading to failure of the FTRD performance.

2. Histological margin: R0 resection was defined as histologically-complete resection with negative lateral and deep resection margins. $\mathrm{R} 1$ resection was defined as histologically incomplete resection with microscopic residual pathology at resection margins, and Rx resection was defined as indeterminate histological margins when the resection margins could not be adequately examined by pathologist. Histopathological examination of the resected specimens was performed locally at each study center. Subgroup analysis for primary endpoints was performed to evaluate technical success and histological resection margins in a subset of patients with only subepithelial lesions (SEL).

Secondary endpoints of this study were:

1. immediate and delayed procedure-related adverse events (as defined below),

2. procedure time, and

3. risk of residual or recurrent lesions on follow-up endoscopy

Immediate adverse events (AEs) were defined as those which occurred and were diagnosed before finishing the index endoscopy session, corresponding to intra-procedure AE in the ASGE lexicon [11]. Any AE that occurred or was diagnosed afterwards was defined as a delayed $A E$, corresponding to post-procedure and late AEs in the ASGE lexicon. Minor immediate bleeding was defined as bleeding that required no intervention beyond endoscopic hemostasis, and major immediate bleeding was defined as bleeding that required any additional intervention beyond endoscopic hemostasis (i. e. blood or blood product transfusion, vasopressors, prolonged admission, and treatment by interventional radiology or surgery). Minor delayed bleeding was defined as bleeding that required no intervention beyond observation and stopped spontaneously, and major delayed bleeding was defined as bleeding that required any intervention (i.e. repeat endoscopy for hemostasis, blood or blood product transfusion, vasopressors, prolonged admission, and treatment by interventional radiology or surgery). Minor bleeding corresponded to a mild $A E$, and major bleeding a moderate to severe $A E$ in the ASGE lexicon. Data were also collected on other AEs including perforation, iatrogenic stricture, injury to adjacent organs, leakage, infection (e.g. peritonitis, bacteremia), or need for surgery. For patients with follow up, endoscopy data were collected on the duration of follow-up, presence of residual or recurrent lesions, pathology results, and need for repeat intervention.

\section{Data management and statistical analysis}

A universal data collection instrument including detailed patient and lesion characteristics as well as outcome definitions was designed at the study coordinating centers (New YorkPresbyterian Hospital, Weill Cornell Medicine, New York, and Johns Hopkins Hospital, Baltimore, Maryland, United States) and was sent to collaborators to ensure consistency across study centers. De-identified patient data were collected using these instruments, and analyzed centrally (NewYork-Presbyterian Hospital, Weill Cornell Medicine, New York). Data were collected on patient demographics, their pre-procedure American Society of Anesthesiologists physical status classification (ASA class) [12], Charlson comorbidity index (CCI) [13], and use of steroids and immunosuppressive and antithrombotic medications. Total procedure time was measured from the insertion of the first endoscope until the withdrawal of the last endoscope (more than one endoscope was needed in most cases). Summary statistics are presented as mean (SD), and median $(I Q R)$, and Chi-squared test, Fisher's exact test, or logistic regression was used to analyze categorical data. All tests are 2tailed with an alpha of 0.05 .

\section{Full-Thickness Resection Device (FTRD)}

The FTRD (Ovesco Endoscopy, Tübingen, Germany) is an integrated system originally designed and approved for full-thickness resection in the colon, which consists of a transparent cap (outer diameter of $21 \mathrm{~mm}$ ) with a modified OTSC provided with a tissue grasper and an integrated hot snare located at the rim of the transparent cap with the handle of the snare running on the outer surface of the endoscope under a transparent plastic sheath. This system can be used with flexible endoscopes with a diameter of 11.5 to $13.2 \mathrm{~mm}$ and a working channel diameter of at least $3.2 \mathrm{~mm}$. After marking the target lesion with the electrocautery probe included in the FTRD kit, the endoscope is removed from the patient, and the FTRD is mounted on an endoscope (a small-caliber colonoscope or a double-channel therapeutic endoscope). In many cases, and as judged necessary by the endoscopist according to the patient's anatomy, bougie or balloon dilation of the esophagus especially at the lower esophageal sphincter in preparation for subsequent passage of the large device cap $(21 \mathrm{~mm}$, or $60 \mathrm{Fr})$ is performed before the endoscope mounted with FTRD is reinserted and advanced to the target lesion. The snare is then connected to an electrosurgical generator. Subsequently the target lesion is grasped using 

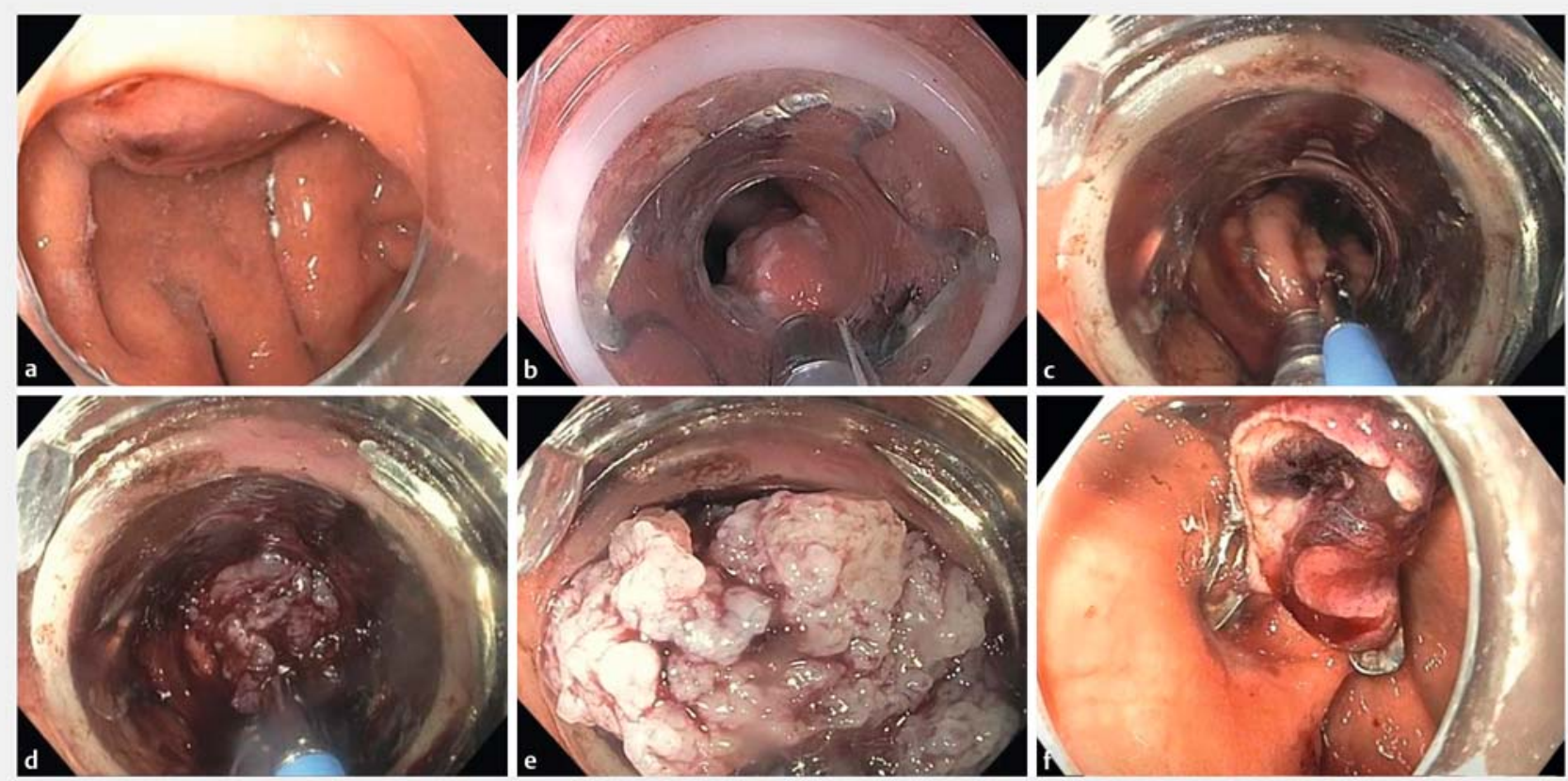

- Fig. 1 Endoscopic full-thickness resection of $13 \mathrm{~mm}$ gastrointestinal stromal tumor using Full-Thickness Resection Device (FTRD). a Initially, a gastroscope fitted with a transparent cap was advanced to the stomach for examining the lesion. $\mathbf{b}$ After marking the margins of the lesions using FTRD marking probe, the gastroscope was removed and replaced with a double-channel therapeutic endoscope fitted with the FTRD device and advanced back to the gastric fundus. c,d Using the FTRD tissue grasper, the submucosal lesion was carefully grasped and pulled into the transparent cap until the marked edges are visualized within the cap. e The integrated over-the-scope clip is then deployed at the base of the target lesion and the integrated snare is immediately closed just above the clip and the resection is completed by applying cutting current. $\mathbf{f}$ Endoscopic view of the resection site and the deployed clip following the FTRD resection.

the tissue grasper forceps and retracted into the transparent cap where the tissue grasper remains fixed in position. After the OTSC clip is deployed at the base of the target lesion and the integrated snare is immediately closed just above the clip, a cutting current is applied to complete the resection ( $\vee$ Fig. $\mathbf{1}$, - Video 1). Finally, the endoscope is removed with the resected specimen within the transparent cap and the specimen is then pinned out on a mounting board and fixed in formaldehyde. An endoscope is then reinserted to examine the site of the resection for signs of incomplete resection or complications such as bleeding, or perforation. Hospital admission after the procedure was at the discretion of the endoscopist.

\section{Results}

\section{Patient and lesion characteristics}

Between January 2017 and February 2019, a total of 56 patients underwent FTRD resection for UGI tract lesions across 13 study centers and were included ( $\vee$ Table 1 ). Patients had a mean age of $61 \pm 14$ years, and $82 \%$ of them were ASA class 1 or $2(\triangleright$ Table 2). Ten patients were on antithrombotics before the procedure: six patients on aspirin, two patients on warfarin, and one patient each on a P2Y12 inhibitor, and dual antiplatelet therapy. Aspirin was continued for four patients but was stopped in two before the procedure, and the remaining antithrombotic medications were held before the procedure. One patient each was on systemic steroids and immunosuppressive therapy be-

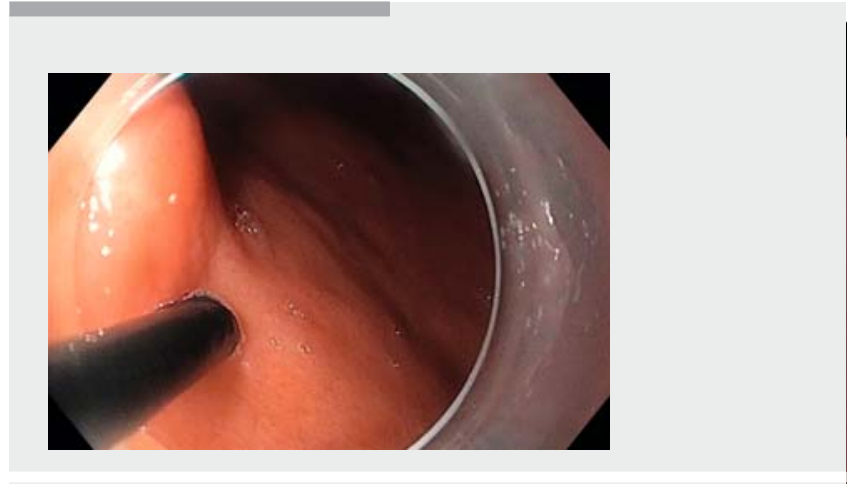

$\nabla$ Video 1 Endoscopic full-thickness resection using FTRD

fore the procedure. Patients had a mean $\mathrm{CCl}$ of 3.5 before the procedure with four ( $7 \%$ ) having a diagnosis of end-stage renal disease, two patients with cirrhosis, ten $(18 \%)$ patients with diabetes, seven (13\%) with congestive heart failure and coronary artery disease, and nine (16\%) with malignancy.

The majority of the FTRDs procedures were performed in the gastric antrum ( $n=21,38 \%$ ) followed by the gastric body ( $n=$ $15,27 \%)$, gastric cardia and fundus ( $n=11,19 \%)$, duodenum $(n=8,14 \%)$, and esophagus $(n=1,2 \%)$. The most common pre-procedural indications ( $\triangleright$ Table 3 ) were initial resection of mesenchymal neoplasms including gastrointestinal stromal tumor, GIST, $(n=23,41 \%)$, diagnostic full-thickness biopsy $(n=$ 
- Table 1 Distribution of FTRD procedures across study centers.

\begin{tabular}{|l|c|}
\hline Study center & FTRD, $\mathbf{n = 5 6}$ \\
\hline Center 1: Ulm, Germany & $15(27)$ \\
\hline Center 2: Freiburg, Germany & $14(25)$ \\
\hline Center 3: Seattle, USA & $5(9)$ \\
\hline Center 4: Baltimore, USA & $4(7)$ \\
\hline Center 5: Danville, USA & $3(5)$ \\
\hline Center 6: Irvine, USA & $3(5)$ \\
\hline Center 7: Jackson, USA & $3(5)$ \\
\hline Center 8: New York, USA & $2(4)$ \\
\hline Center 9: Zurich, Switzerland & $2(4)$ \\
\hline Center 10: Seattle, USA & $2(4)$ \\
\hline Center 11: New York, USA & $1(2)$ \\
\hline Center 12: Scottsdale, USA & $1(2)$ \\
\hline Center 13: Tübingen, Germany & $1(2)$ \\
\hline FTRD, full-thickness resection device & \\
\hline
\end{tabular}

- Table 2 Patient and lesion characteristics of FTRD for upper gastrointestinal tract lesions.

\begin{tabular}{|l|c|}
\hline Total number of FTRDs & 56 \\
\hline Age, mean (SD) & $61(14)$ \\
\hline Female, $\mathrm{n}(\%)$ & $26(46)$ \\
\hline ASA category, $\mathrm{n}(\%)$ & $18(32)$ \\
\hline - 1 & $28(50)$ \\
\hline - 2 & $9(16)$ \\
\hline - 3 & $1(2)$ \\
\hline - 4 & $3.5(0-9)$ \\
\hline Charlson comorbiditiy index, mean (range) & \\
\hline Antithrombotic use ${ }^{1}, \mathrm{n}(\%)$ & $46(82)$ \\
\hline - None & $6(10)$ \\
\hline - Aspirin & $1(2)$ \\
\hline - P2Y12 inhibitor & $1(2)$ \\
\hline - Aspirin plus P2Y12 inhibitor & $2(4)$ \\
\hline - Warfarin & $11(27)$ \\
\hline FTRD location, $\mathrm{n}(\%)$ & $1(2)$ \\
\hline - Esophagus & $119)$ \\
\hline - Cardia/Fundus & \\
\hline - Stomach body & \\
\hline - Antrum & Duodenum \\
\hline
\end{tabular}

\section{- Table 2 (Continuation)}

Endosonographic layer of origin, $\mathrm{n}(\%)$

- Mucosa

- Submucosa

- Muscularis Propria

- Unknown

Previous intervention, $\mathrm{n}(\%)$

- None

- Biopsy

- Hot snare resection

- EMR

- ESD

Result of previous intervention, $\mathrm{n}(\%)$

- Partial resection with positive macroscopic margin

- Complete resection with positive microscopic margin

- Failed prior resection

- Complete resection with recurrence

- No previous attempt at resection

Non-lifting sign, $\mathrm{n}(\%)$

- Not tested

- Negative, could be completely lifted

- Positive, could not be lifted

FTRD, full-thickness resection device; EMR, endoscopic mucosal resection; ESD, endoscopic submucosal dissection

${ }^{1}$ Aspirin was stopped for two patients before the procedure; P2Y12 inhibitor and Warfarin were stopped before the procedure.

$10,18 \%)$, initial resection of adenocarcinoma $(n=5,9 \%)$, initial resection of neuroendocrine tumor $(n=5,9 \%)$, resection of residual or recurrent adenoma $(n=4,7 \%)$, resection of residual or recurrent neuroendocrine tumor $(n=4,7 \%)$, resection of residual or recurrent mesenchymal neoplasms including GIST ( $\mathrm{n}=$ $3,5 \%)$, and initial resection of adenoma $(n=2,4 \%)$. For $39 \%$ of lesions the index FTRD resection was the first diagnostic or therapeutic intervention, and for $78 \%$ of the lesions it was the first therapeutic intervention ( $\downarrow$ Table 2 ). Twenty-two lesions (39\%) were biopsied before FTRD resection. Four patients with pre-procedure diagnosis of recurrent or residual neuroendocrine tumor had undergone EMR or ESD (complete endoscopic resection but positive histological margins reported in all cases) between 3 and 5 months before the FTRD procedure and the final pathologies after FTRD resection showed normal or scar tissue. Three patients with pre-procedure diagnosis of residual adenomas had undergone partial resection (hot snare, EMR, and ESD) between one and three months before FTRD resection and their final pathologies showed tubular adenoma. Two patients with pre-procedure diagnoses of residual mesenchymal 
- Table 3 Pre-procedure indication, final pathology, and prior treatment history of the lesions.

\begin{tabular}{|c|c|c|}
\hline Pre-procedure indication & Final Pathology & Prior treatment \\
\hline Mesenchymal neoplasm including GIST, initial resection, $n=23$ & $\begin{array}{l}\text { Mesenchymal neoplasm, other than GIST, } n=10 \\
\text { GIST, } n=7 \\
\text { Hamartomatous polyp, } n=2 \\
\text { Ectopic pancreas, } n=2 \\
\text { Normal tissue, } n=1 \\
\text { Unavailable, } n=1\end{array}$ & NA \\
\hline \multirow[t]{6}{*}{ Full-thickness biopsy, $\mathrm{n}=10$} & Hamartomatous polyp, $\mathrm{n}=4$ & \\
\hline & GIST, $n=2$ & \\
\hline & Adenoma with $\mathrm{HGD}, \mathrm{n}=1$ & \\
\hline & Hyperplastic polyp, $\mathrm{n}=1$ & \\
\hline & Mesenchymal neoplasm, other than GIST, $n=1$ & \\
\hline & Scar tissue, $n=1$ & EMR \\
\hline Adenocarcinoma, intial resection, $\mathrm{n}=5$ & $\begin{array}{l}\text { Adenocarcinoma, } \mathrm{n}=3 \\
\text { Tubular adenoma, } \mathrm{n}=1 \\
\text { Scar tissue }^{1}, \mathrm{n}=1\end{array}$ & $N A^{1}$ \\
\hline NET, initial resection, $n=5$ & $\begin{array}{l}\text { NET, } n=4 \\
\text { Hyperplastic polyp, } n=1\end{array}$ & NA \\
\hline NET, residual, $n=4$ & Normal or scar tissue, $\mathrm{n}=4$ & EMR, ESD \\
\hline \multirow[t]{2}{*}{ Adenoma, residual, $\mathrm{n}=4$} & Tubular adenoma, $\mathrm{n}=3$ & Hot snare, EMR, ESD \\
\hline & Scar tissue, $\mathrm{n}=1$ & EMR \\
\hline Mesenchymal neoplasm including GIST, residual or recurrent, $n=3$ & Mesenchymal neoplasm, other than GIST, $n=3$ & EMR, Cap-assisted EMR \\
\hline Adenoma, initial resection, $\mathrm{n}=2$ & $\begin{array}{l}\text { Tubular adenoma, } n=1 \\
\text { Sessile Serrated Adenoma, } n=1\end{array}$ & NA \\
\hline \multicolumn{3}{|c|}{$\begin{array}{l}\text { GIST, gastrointestinal stromal tumor; FTRD, full-thickness resection device; NET, neuroendocrine tumor; EMR, endoscopic mucosal resection; ESD, endoscopic sub- } \\
\text { mucosal dissection } \\
1 \text { Patient underwent a biopsy with jumbo forceps before the FTRD, which removed the adenocarcinoma completely, and the final FTRD pathology showed only scar } \\
\text { tissue with surrounding intestinal metaplasia. }\end{array}$} \\
\hline
\end{tabular}

neoplasm had undergone partial resection with EMR and capassisted EMR 2 to 4 years before the FTRD resection and their final pathologies revealed mesenchymal neoplasm ( $\downarrow$ Table 3 ,

- Fig. 2).

Mean lesion size estimated before FTRD was $12 \pm 4 \mathrm{~mm}$ with a range of $3 \mathrm{~mm}$ to maximum of $20 \mathrm{~mm}$. Endoscopic Ultrasound (EUS) was performed for 53 (95\%) of lesions before resection and showed the layer of origin to be submucosa, muscularis propria, and mucosa in $41 \%, 31 \%$, and $23 \%$ of the lesions, respectively. Lifting of the lesion with submucosal injection was attempted in only seven lesions before resection and five of them could not be lifted.

\section{Outcomes}

\section{Technical success}

FTRD resection led to complete technical success in 43 patients (77\%), and partial success in another nine patients (16\%). There were two incomplete procedures (4\%): The target lesion could not be reached with the FTRD device for a GIST in the gastric fundus, and for a GIST in the antrum the target lesion was reached but the FTRD device was not deployed as the lesion could not be retracted into the cap ( $\vee$ Table 3 ; $\triangleright$ Table 4 ). There were two cases of device failure. In both the integrated snare snapped after deployment of the clip, and the lesion was resected above the clip with either a separate snare or an endoscopic knife. Of the nine patients who had partial FTRD resection, four underwent additional endoscopic intervention during the same session with forceps avulsion and hot snare resection.

There was a trend toward association between the size of the FTRD resection specimen and technical success rate as procedures that led to the resection of a larger specimen were more likely to achieve complete technical success (OR 1.12, $95 \% \mathrm{Cl}$ $0.99-1.25, P=0.05)$. However, the size of the target lesion itself was not significantly associated with technical success rate $(P>$ $0.05)$. The number of FTRD procedures performed in an institution was not significantly associated with the technical success rate $(P=0.827)$. Lesion location, layer of origin, Paris classification, pathology, or previous intervention were not associated with the technical success rate $(P>0.05)$.

The majority (62\%) of FTRD procedures were performed under propofol sedation, $36 \%$ with general anesthesia, and $2 \%$ under conscious sedation. The majority of procedures $(n=29$, $52 \%$ ) were performed without antibiotic prophylaxis, while 21 
Initial indication for FTRD versus final pathology

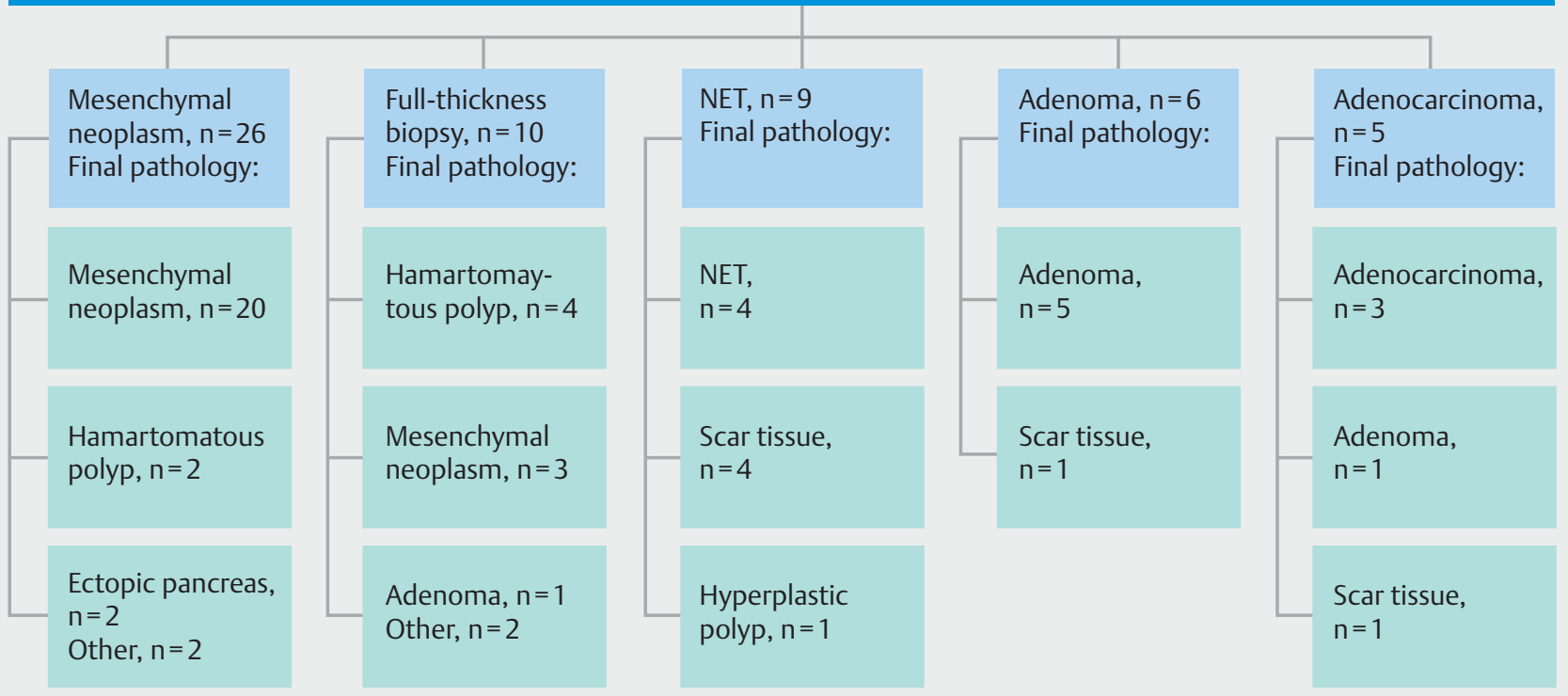

- Fig. 2 Association between indication and final pathology of upper gastrointestinal tract FTRD resection.

\begin{tabular}{|c|c|c|}
\hline Technical success ${ }^{1}$ & $N=56$ & Description \\
\hline Complete success & $n=43$ & complete en bloc endoscopic resection \\
\hline Partial success & $\mathrm{n}=9$ & partial endoscopic resection with positive macroscopic margins \\
\hline \multirow[t]{2}{*}{ Incomplete procedure } & \multirow[t]{2}{*}{$\mathrm{n}=2$} & GIST in gastric fundus: The target lesion could not be reached with the FTRD device \\
\hline & & GIST in the antrum: the lesion could not be retracted into the cap \\
\hline Device failure & $n=2$ & $\begin{array}{l}\text { For both: the integrated snare snapped after deployment of the clip, and the lesion was resected above the } \\
\text { clip with a separate instrument }\end{array}$ \\
\hline Histological margin & $N=56$ & Description \\
\hline RO & $n=38$ & Histologically-complete resection with negative lateral and deep resection margins \\
\hline \multirow[t]{2}{*}{ R1 } & \multirow[t]{2}{*}{$n=10$} & 4 cases of complete en bloc resection with positive lateral margins \\
\hline & & 6 cases of partial resection with positive lateral margins \\
\hline \multirow[t]{2}{*}{ Rx } & \multirow[t]{2}{*}{$\mathrm{n}=2$} & 1 case of complete en bloc resection with indeterminate margins \\
\hline & & 1 case of partial resection with indeterminate margins \\
\hline Missing & $n=2$ & 2 cases for whom data for the histological margin were missing \\
\hline Not applicable & $n=4$ & 4 cases of incomplete procedure or device failure described above, without any tissue resection \\
\hline \multicolumn{3}{|c|}{$\begin{array}{l}\text { GIST, gastrointestinal stromal tumor; FTRD, full-thickness resection device } \\
1 \text { Complete technical success: defined as reaching the target lesion with the FTRD, correct application of the FTRD clip on the lesion and immediate resection with the } \\
\text { integrated snare AND complete en bloc endoscopic resection. Partial success was defined as reaching the target lesion with the FTRD, correct application of the } \\
\text { FTRD clip on the lesion and immediate resection with the integrated snare but partial endoscopic resection with positive macroscopic margins Procedure was } \\
\text { considered incomplete if the target lesion could not be reached with the FTRD device or if the target lesion was reached but the endoscopist decided against de- } \\
\text { ploying the FTRD due to lesion characteristics. Device failure was defined as over-the-scope clip, tissue grasper, or integrated snare malfunction in any way leading } \\
\text { to failure of the FTRD. RO resection was defined as histologically-complete resection with negative lateral and deep resection margins. R1 resection was defined as } \\
\text { histologically incomplete resection with microscopic residual pathology at resection margins, and Rx resection was defined as indeterminate histological margins } \\
\text { when the resection margins could not be adeguately examined by pathologist. }\end{array}$} \\
\hline
\end{tabular}


patients $(37 \%)$ received a single intravenous dose of pre-procedural antibiotic, and six (11\%) received combined pre- and post-procedural prophylactic antibiotics. Mean total procedure time was $42 \pm 16$ minutes with a range of 18 to 90 minutes.

\section{Histological margin of resection}

Of the 43 patients with complete technical success, 38 (88\%) had negative histological margins indicating an $\mathrm{R} 0$ resection yielding an overall rate of $68 \%$ R0 resections of all the performed FTRD resections. Four (10\%) patients with complete en bloc resection had $\mathrm{R} 1$ resection with positive histological margin, and one $(2 \%)$ had an indeterminate $(R x)$ resection margin. Of the nine patients with partial technical success, six had $\mathrm{R} 1$ resection with positive histological margin, one had an Rx or indeterminate resection margin, and the data for the histological margin for other two patients was missing ( $>$ Table 4$)$. The mean diameter of resected specimen was $15 \pm 9 \mathrm{~mm}$, ranging from $3 \mathrm{~mm}$ to $35 \mathrm{~mm}$, with $10 \%$ of the resected specimens having a diameter of $30 \mathrm{~mm}$ or greater on pathologic exam. The mean depth of resected specimen was $6 \pm 4 \mathrm{~mm}$ ranging from $2 \mathrm{~mm}$ to a maximum $18 \mathrm{~mm}$ with $10 \%$ of the resected specimens having a depth of $14 \mathrm{~mm}$ or greater on pathologic exam ( $\triangleright$ Table 5). Lesions size was inversely associated with probability of successful R0 resection $(\mathrm{OR}=0.85,95 \% \mathrm{Cl} 0.72-0.99, P=$ 0.038 ). In fact, the probability of complete R0 resection changed from $73 \%$ in lesions with a diameter $<15 \mathrm{~mm}$ to only $29 \%$ in lesions with a diameter $\geq 15 \mathrm{~mm}(P=0.034)$.

\section{Subgroup analysis}

Subgroup analysis was performed including only the subepithelial lesions (SELs) to evaluate the technical success rate and histological margin of resection in lesions arising from submucosa and muscularis propria. There were 27 SELs including Neuroendocrine tumors, granular cell tumors, and mesenchymal neoplasms including GIST. Thirty-six percent of SELs originated from muscularis propria, and the remaining $64 \%$ originated from submucosa. FTRD resection led to complete technical success in 21 patients (78\%) with SEL, and partial success in another three patients (11\%). There were two incomplete procedures (7.5\%) for two gastric GISTs, as described above. There was one case of device failure due to malfunction the integrated snare as described above. The complete success rate did not differ significantly between SELs (78\%), and the rest of the of the lesions ( $73 \%, P=0.643$ ). Of the 27 patients with SEL, 19 (70\%) had negative histological margins indicating an $\mathrm{R} 0$ resection. Five (19\%) patients had $\mathrm{R} 1$ resection with positive histological margin. The R0 resection rate did not differ significantly between SELs (70\%), and the rest of the of the lesions (66\%, $P$ $=0.698)$. The mean diameter of resected SEL specimens was 16 $\pm 8 \mathrm{~mm}$, ranging from $6 \mathrm{~mm}$ to $35 \mathrm{~mm}$, with $10 \%$ of the resected specimens having a diameter of $30 \mathrm{~mm}$ or greater on pathologic exam. Mean depth of resected SEL specimens was $9 \pm 4 \mathrm{~mm}$ ranging from $5 \mathrm{~mm}$ to a maximum $18 \mathrm{~mm}$ with $10 \%$ of the resected specimens having a depth $\geq 15 \mathrm{~mm}$ on pathologic exam. Mean depth of the resected specimens was larger for SELs compared to the mucosal lesions ( $9 \mathrm{~mm}$ versus $4 \mathrm{~mm}, P<0.001$ ). Similar to the overall analysis, the size of the lesion was inverse-
- Table 5 Procedural details and outcomes of FTRD for upper gastrointestinal tract lesions.

Total number of FTRDs 56

Antibiotic prophylaxis, $\mathrm{n}(\%)$

- None

- Single-dose pre-procedural

- Pre and post-procedural

Sedation, $\mathrm{n}(\%)$

- Propofol procedural anesthesia

$35(62)$

- General anesthesia $20(36)$

- Conscious sedation

Procedure time; minutes, mean (range)

$42(18-90)$

Diameter of resected lesion (mm), mean (SD)

16 (9)

Diameter of resected lesion $(\mathrm{mm})$, range

3-35

Depth of resected lesion (mm), mean (SD)

Depth of resected lesion $(\mathrm{mm})$, range

Technical success ${ }^{1}, \mathrm{n}(\%)$

- Complete success $43(77)$

- Partial success 9 (16)

- Incomplete procedure

- Device failure

Histological margin, $n$ (\%)

- R0 $38(68)$

- $\mathrm{R} 1$ $10(17)$

- $\mathrm{Rx}$ $2(4)$

- Missing $2(4)$

- NA (incomplete or failed procedure)

Complications, $\mathrm{n}(\%)$

- Minor intraprocedural bleeding $4(7)$

- Major intraprocedural bleeding

- Minor delayed bleeding $2(4)$

- Major delayed bleeding

- Injury, including adjacent organs

${ }^{1}$ Technical success: defined as reaching the target lesion with the FTRD, correct application of the FTRD clip on the lesion and immediate resection with the integrated snare AND complete en bloc endoscopic resection. Partial success was defined as reaching the target lesion with the FTRD, correct application of the FTRD clip on the lesion and immediate resection with the integrated snare but partial endoscopic resection with positive macroscopic margins Procedure was considered incomplete if the target lesion could not be reached with the FTRD device or if the target lesion was reached but the endoscopist decided against deploying the FTRD due to lesion characteristics. Device failure was defined as over-the-scope clip, tissue grasper, or integrated snare malfunction in any way leading to failure of the FTRD 
ly associated with the probability of successful $R 0$ resection $(\mathrm{OR}=0.67,95 \% \mathrm{Cl} 0.48-0.98, P=0.035)$ in patients with $\mathrm{SEL}$.

\section{Adverse events}

There were a total of $12(21 \%)$ reported AEs. Their severity based on ASGE Lexicon were classified as mild in seven patients $(12 \%)$, and moderate in five patients $(9 \%)$, without any reported severe AEs [11].

Immediate intraprocedural AEs were reported in nine patients (16\%) (> Table 5): four cases (7\%) of immediate minor bleeding (mild $A E$ ), one managed conservatively and three treated with thermal coagulation; four cases (7\%) of immediate major bleeding needing transfusion and/or pressors (moderate $\mathrm{AE}$ ), one managed with thermal coagulation while the remaining three with hemostatic clips; and one case of mucosal/submucosal injury by the integrated snare to the contralateral luminal wall at gastroesophageal junction that did not result in perforation or bleeding and was managed conservatively with two days of observation in hospital (mild AE).

Delayed AEs were reported in three cases: two cases of minor bleeding 1 and 7 days after the procedure, both managed conservatively (mild $\mathrm{AE}$ ), and one case of delayed major bleeding 2 days after the procedure which was managed endoscopically without need for surgery (moderate AE).

Four patients were taking aspirin at the time of the procedure, but there was no association between aspirin use and risk of bleeding $(P=0.470)$. Similarly, size of lesion, previous biopsy or attempted resection, procedure length, and pathology of the resected lesion or its layer of origin were not associated with bleeding $(P>0.05)$.

A majority $(70 \%)$ of the patients were admitted following the procedure for a median of 3 days after FTRD resection (IQR 2-3 days) with a range of 1 to 6 days post-procedure admission, while 17 patients (30\%) were discharged on the day of the procedure. The majority (73\%) of patients who were admitted post-procedurally did not have any complications and there was a strong association between study centers and admitting patterns with some centers admitting all of their FTRD resection patients for observation and some centers discharging patients on the same day. After accounting for the effect of institutions on admission patterns after the FTRD procedure, complications (early or late) were associated with an increased chance of admission $(P=0.05)$ but none of the other variables including previous treatment, location, size, layer of origin, Paris classification, indication or final pathology were associated with post-procedural admission $(P>0.05)$.

\section{Follow-up}

Patients were followed for a median of 3 months (IQR 1-6 months) after the procedure. There was one death reported 49 days after the procedure due to underlying end-stage heart failure, deemed not to be related to the procedure itself. Follow-up endoscopy was accomplished in 31 patients (55\%), on average 88 days after FTRD resection (IQR 68-138 days). The majority of these patients $(n=30,97 \%)$ did not have any residual or recurrent lesion on endoscopic examination and biopsy. Residual adenoma was found only in one patient who had an in- itial partial resection of tubular adenoma and was treated successfully with endoscopic resection. In $43 \%$ the FTRD clip was still in place during the follow up endoscopy and three patients had mucosal ulceration at the site of the resection when examined during the follow up endoscopy without need for endoscopic therapy.

\section{Discussion}

This study reports indications, outcomes, and safety of the FTRD for endoscopic full-thickness resection (EFTR) of UGI tract lesions, using data from early-adopters of this technique in Europe and United States. The most frequent indication for use of the FTRD in this cohort was resection of mesenchymal neoplasms, including GIST, most commonly located in the stomach. Although the FTRD is currently approved only for use in the lower gastrointestinal tract lesions, our results suggest an acceptable technical and clinical success rate and safety profile for using FTRD in the foregut, making it a potential alternative to other resection techniques such as ESD.

Previous studies have evaluated the FTRD for resection of colonic lesions. In the most comprehensive prospective report to date, Schmidt et al reported the results of FTRD resection in 181 patients with colonic lesions including subepithelial lesions (SEL). They reported a technical success rate of $89.5 \%$ with complete en bloc resection, and an R0 resection rate of $76.9 \%$. The AE rate was reported at $9.9 \%$ with $2.2 \%$ need for emergency surgery, and recurrent/residual tumor seen in $15.3 \%$ of cases at 3 months' follow-up [5]. They reported bleeding as a moderate $A E$ in four (2.2\%), all of which happened after the index FTRD session (Days 1 to 3 ), and were managed endoscopically without need for surgery. They also reported perforation in six (3.3\%), five of which occurred because the clip was not deployed properly leading to an immediate perforation. In contrast, reports on use of the FTRD device for resection of UGI tract lesions are more limited. In the largest case series using the FTRD device for resection of duodenal lesions, Bauder et al. reported a series of 20 patients ( 13 adenomas, and 5 SELs), with an $85 \%$ technical success rate and overall $\mathrm{R} 0$ resection rate of $60 \%$. They reported minor bleeding rate of $16 \%$, but no major bleeding or perforations [10]. Compared with these results, we report a technical success rate of $77 \%$ and an $\mathrm{R} 0$ resection rate of $68 \%$, comparable to the previously reported results in the duodenum. Similarly, there were no perforations in our study, and the most common adverse event was mild or moderate procedure-related bleeding with a rate of $19.6 \%$, all managed successfully either conservatively or endoscopically. It should be noted that although the size of lesions in our report is similar to the above-mentioned series of duodenal lesions (average of 16 vs $17 \mathrm{~mm}$ ), the type of lesions is different with SELs comprising $72 \%$ of lesions in our report compared with only $25 \%$ in the report by Bauder et al, possibly accounting for the difference in the en bloc resection rates. More recently, Meier et al. (RESECT trial) have reported results of using a modified version of the FTRD (Gastroduodenal or gFTRD, Ovesco Endoscopy, Tübingen, Germany) for resection of gastric SELs in 29 patients [14]. Compared with the colonic FTRD device, gFTRD has 
a slightly narrower cap (19.5 vs $21 \mathrm{~mm}$ for colonic FTRD) and can be used with endoscopes with a smaller diameter (10.5 vs $11.5 \mathrm{~mm}$ for colonic FTRD).

Furthermore, the device comes with an insertion balloon to facilitate its passage through the UGI tract. This prospective study was limited to gastric SELs $\leq 15 \mathrm{~mm}$ (median lesion size $11 \mathrm{~mm}$ ) and reports a technical success rate of $89.7 \%$ for en bloc resection, and $\mathrm{R} 0$ resection rate of $76 \%$, with immediate minor bleeding as the only observed complication seen in $31 \%$ of patients, which was managed endoscopically in all cases. Given the significant difference in lesion size (median of 11 vs $17 \mathrm{~mm}$ in our study), it is unclear how much of the observed difference in the technical success and $\mathrm{R} 0$ resection rates $(89.5 \%$ vs $77 \%$ and $76 \%$ vs $68 \%$, respectively) is attributable to the design of the new device or expertise of the study centers. Combining the above results, the available data suggest that using FTRD for resection of UGI tract lesions, including SELs, can lead to similar success rates compared with colonic FTRD, while potentially associated with lower rate of severe AEs, given that the most common, and almost exclusive, reported $A E$ has been mild to moderate procedure-related bleeding. The higher rate of immediate mild bleeding but fewer perforations compared with significantly lower rates of immediate bleeding but higher perforation rate for FTRD in the colon is an interesting finding [5]. This might be explained on the basis of thicker wall and better blood supply of the stomach compared to the colon with a protective effect against perforation but increasing the likelihood of procedure-related bleeding.

In our cohort, the most common final pathology was mesenchymal neoplasms including GIST ( $n=23,41 \%$ of lesions), mainly located in the stomach, which is compatible with the known epidemiology of gastric GIST as the most common subepithelial lesion (SEL) in the GI tract [15]. Although all GIST have some malignant potential, only about one fourth of gastric GISTs are malignant at the time of diagnosis [16]. Available diagnostic and prognostic tools such as size and location of the lesion, endoscopic findings, EUS findings and results of EUSguided biopsy can help make accurate diagnosis and risk stratify before decision for resection is made for SELs of upper gastrointestinal tract, but these tools have suboptimal accuracy for mesenchymal neoplasms including GIST, as well as for SELs in general [17-21]. Therefore, considering the potentially limited histological data available from conventional, "bite-onbite," or even EUS-guided biopsies [22], en bloc resection of UGI tract SELs represents the most accurate diagnostic tool as well as a potentially curative treatment, especially as some guidelines suggest resection even for small GISTs (i.e. $<2 \mathrm{~cm}$ ) as there is a potential for progression and metastasis [23].

Although there is a lack of direct comparison with surgical resection, endoscopic resection of UGI tract SELs, while remaining a controversial option, has been shown to be effective and safe $[24,25]$. Submucosal dissection and resection including ESD and STER can achieve en bloc endoscopic resection of SELs. However, compared with FTRD, they are more technically challenging, more time consuming, and at least in the case of ESD, associated with high risks of complications such as perforation. For ESD of gastric SELs, perforation rates as high as
$14 \%$ have been reported [24] and pooled analysis of the STER case series has showed a perforation rate of $6 \%$ and high rates of air-leakage [26]. In comparison, there were no cases of perforation in our cohort or other case series of UGI tract FTRD for resection of UGI tract SELs. It should be noted however that almost all of the reported complications with ESD and STER are managed successfully either during the index endoscopic session or conservatively afterwards, and do not seem to affect the final outcome of the procedure. Additionally, the submucosal dissection techniques have the advantage of being able to treat larger lesions, as FTRD is limited by the size of the device cap and clip [27].

The effectiveness of using FTRD depends on the ability of positioning the lesion completely within the device cap prior to deploying the clip. This in turn depends on lesion size and mobility, with the latter influenced mostly by anatomic location and presence of submucosal fibrosis. In our cohort, the largest size of the resected specimen was $35 \mathrm{~mm}$ and size of the target lesion or its location even after multiple sensitivity analyses for separating duodenal and gastric lesions were not found to be significantly associated with technical success or R0 resection rates. Similarly, we did not find an association between history of prior endoscopic intervention and FTRD outcome. Another commonly encountered limitation of the FTRD is the size of the device cap $(21 \mathrm{~mm})$ which makes traversing the upper and lower esophageal sphincters and pylorus difficult in some cases. Many endoscopists use bougie or balloon dilation immediately before passage of the FTRD. In certain cases, the relatively limited flexibility of the device mounted on larger endoscopes (typically a slim colonoscope) can also hinder reaching the target lesion as was the case for a patient in our study in which the endoscopist was not able to reach the target lesions located in the fundus.

This study is limited due to its retrospective design with a limited sample size, leading to possible selection bias and potential for limited power and type II error when assessing the above presented correlations between different variables and FTRD outcomes. We also did not have data for follow-up endoscopy for all the cases, limiting assessment of long-term FTRD resection outcomes. Therefore, our estimates of recurrence should be interpreted cautiously. Compared to other available reports, we were able to gather data on a larger number of patients and from a variety of early users of the UGI tract FTRD resection technique from different centers in the Unites States and Europe to have a more representative sample of the patient population and a more accurate estimate on "real-world" FTRD outcomes and complications.

\section{Conclusion}

Our results suggest that FTRD is a relatively safe and effective option for endoscopic resection of appropriate UGI tract lesions including SELs, when compared with other available methods. Two-thirds of our patients had complete histological resection. Although $21 \%$ of patients experienced mild to moderate AEs, there were no severe or fatal AEs. Endoscopists should carefully consider different endoluminal resection options based on le- 
sion size and location, and expect to encounter and manage both immediate and delayed procedure-related bleeding when planning to use this device.

\section{Competing interests}

Dr. Khashab is a consultant for Boston Scientific, Medtronic and Olympus. Dr. Grimm is a consultant for Boston Scientific. Dr. Irani is a consultant for Boston Scientific. Dr. Kumbhari is a consultant for ReShape Life Sciences, Apollo Endosurgery, Medtronic, and Boston Scientific. Dr. Nikhil is a consultant for Apollo Endosurgery, Boston scientific, and Olympus. Dr. Amateau is a consultant for Merit Endoscopy, Boston Scientific, US Endoscopy, and Neurotronic and the recipient of research support from Cook Medical. Dr. Smallfield has research funding from CSA Medical and C2 Therapeutics. Dr. Aadam is a consultant for Boston Scientific. Dr. Diehl is a consultant for Boston Scientific, Olympus, Pentax, and Cook Medical. Dr. Chang is a consultant for Apollo, Boston Scientific, Cook, Covidien, Erbe, Endogastric Solutions, Mauna Kea Technologies, Mederi, Medtronic, Olympus, Ovesco, Pentax, and Torax. Dr. Samarasena has an educational grant from Cook and is a consultant for Mauna Kea Technologies, Medtronic, Olympus, Pentax, and US Endoscopy. Dr. Al-Haddad received research and teaching support from Boston Scientific. Dr. Friedland is a consultant for Boston Scientific and CapsoVision. Dr. Templeton is a consultant for Boston Scientific and Medtronic. Dr. Ginsberg is a consultant for Olympus and Boston Scientific. Dr. Fukami is a consultant for Boston Scientific and Olympus. Dr. Mahadev is a consultant for Olympus and Conmed. Dr Sharaiha is a consultant for Olympus, Boston Scientific, and Cook. Dr Carr-Locke is a consultant for Boston Scientific Endoscopy and receives royalties from Steris Corporation and Telemed Systems. Dr. Schmidt has received lecture fees and research funding from Ovesco Endoscopy.

\section{References}

[1] Evans JA, Chandrasekhara V, Chathadi KV et al. The role of endoscopy in the management of premalignant and malignant conditions of the stomach. Gastrointest Endosc 2015; 82: 1-8

[2] Perez A, Saltzman JR, Carr-Locke DL et al. Benign nonampullary duodenal neoplasms. J Gastrointest Surg 2003; 7: 536-541

[3] Chung IK, Lee JH, Lee SH et al. Therapeutic outcomes in 1000 cases of endoscopic submucosal dissection for early gastric neoplasms: Korean ESD Study Group multicenter study. Gastrointest Endosc 2009; 69: 1228-1235

[4] Cao Y, Liao C, Tan A et al. Meta-analysis of endoscopic submucosal dissection versus endoscopic mucosal resection for tumors of the gastrointestinal tract. Endoscopy 2009; 41: 751-757

[5] Schmidt A, Beyna T, Schumacher B et al. Colonoscopic full-thickness resection using an over-the-scope device: a prospective multicentre study in various indications. Gut 2018; 67: 1280-1289

[6] Schmidt A, Bauerfeind P, Gubler C et al. Endoscopic full-thickness resection in the colorectum with a novel over-the-scope device: first experience. Endoscopy 2015; 47: 719-725

[7] Richter-Schrag H], Walker C, Thimme R et al. [Full thickness resection device (FTRD). Experience and outcome for benign neoplasms of the rectum and colon]. Der Chirurg; Zeitschrift fur alle Gebiete der operativen Medizen 2016; 87: 316-325

[8] Fahndrich M, Sandmann M. Endoscopic full-thickness resection for gastrointestinal lesions using the over-the-scope clip system: a case series. Endoscopy 2015; 47: 76-79
[9] Sarker S, Gutierrez JP, Council L et al. Over-the-scope clip-assisted method for resection of full-thickness submucosal lesions of the gastrointestinal tract. Endoscopy 2014; 46: 758-761

[10] Bauder M, Schmidt A, Caca K. Endoscopic full-thickness resection of duodenal lesions-a retrospective analysis of 20 FTRD cases. United Europ Gastroenterol ] 2018; 6: 1015-1021

[11] Cotton PB, Eisen GM, Aabakken L et al. A lexicon for endoscopic adverse events: report of an ASGE workshop. Gastrointest Endosc 2010; 71: 446-454

[12] Doyle DJ, Garmon EH. American Society of Anesthesiologists Classification (ASA Class). In: StatPearls. Treasure Island (FL) 2018

[13] Charlson ME, Pompei P, Ales KL et al. A new method of classifying prognostic comorbidity in longitudinal studies: development and validation. J Chronic Dis 1987; 40: 373-383

[14] Meier B, Schmidt A, Glaser N et al. Endoscopic full-thickness resection of gastric subepithelial tumors with the gFTRD-system: a prospective pilot study (RESET trial). Surg Endosc 2019: doi:10.1007/s00464-01906839-2

[15] Faulx AL, Kothari S, Acosta RD et al. The role of endoscopy in subepithelial lesions of the GI tract. Gastrointest Endosc 2017; 85: 11171132

[16] Miettinen M, Lasota J. Gastrointestinal stromal tumors: pathology and prognosis at different sites. Sem Diagn Pathology 2006; 23: 70-83

[17] Polkowski M. Endoscopic ultrasound and endoscopic ultrasoundguided fine-needle biopsy for the diagnosis of malignant submucosal tumors. Endoscopy 2005; 37: 635-645

[18] Hwang JH, Rulyak SD, Kimmey MB. American Gastroenterological Association Institute technical review on the management of gastric subepithelial masses. Gastroenterology 2006; 130: 2217-2228

[19] Faigel DO, Abulhawa S. Gastrointestinal stromal tumors: the role of the gastroenterologist in diagnosis and risk stratification. J Clin Gastroenterol 2012; 46: 629-636

[20] Lim TW, Choi CW, Kang DH et al. Endoscopic ultrasound without tissue acquisition has poor accuracy for diagnosing gastric subepithelial tumors. Medicine 2016; 95: e5246

[21] Mullady DK, Tan BR. A multidisciplinary approach to the diagnosis and treatment of gastrointestinal stromal tumor. J Clin Gastroenterol 2013; 47: 578-585

[22] Eckardt A], Adler A, Gomes EM et al. Endosonographic large-bore biopsy of gastric subepithelial tumors: a prospective multicenter study. Europ J Gastroenterol Hepatol 2012; 24: 1135-1144

[23] Blackstein ME, Blay JY, Corless C et al. Gastrointestinal stromal tumours: consensus statement on diagnosis and treatment. Can J Gastroenterol 2006; 20: 157-163

[24] He Z, Sun C, Wang J et al. Efficacy and safety of endoscopic submucosal dissection in treating gastric subepithelial tumors originating in the muscularis propria layer: a single-center study of 144 cases. Scand J Gastroenterol 2013; 48: 1466-1473

[25] Zhou PH, Yao LQ, Qin XY et al. Endoscopic full-thickness resection without laparoscopic assistance for gastric submucosal tumors originated from the muscularis propria. Surgical endoscopy 2011; 25: 2926-2931

[26] Lv XH, Wang CH, Xie Y. Efficacy and safety of submucosal tunneling endoscopic resection for upper gastrointestinal submucosal tumors: a systematic review and meta-analysis. Surgical endoscopy 2017; 31 : 49-63

[27] Tan Y, Tang X, Guo T et al. Comparison between submucosal tunneling endoscopic resection and endoscopic full-thickness resection for gastric stromal tumors originating from the muscularis propria layer. Surg Endosc 2017; 31: 3376-3382 